\title{
High-quality genome assemblies of male and female Populus $\mathbf{x}$ sibirica plants
}

\author{
Pushkova E.N. ${ }^{1 *}$, Krasnov G.S. ${ }^{1}$, Dvorianinova E.M. ${ }^{1,2}$, Novakovskiy R.O. ${ }^{1}$, Povkhova L.V. ${ }^{1,2}$, \\ Melnikova N.V. ${ }^{1}$, Dmitriev A.A. ${ }^{1}$ \\ ${ }^{1}$ Engelhardt Institute of Molecular Biology, Russian Academy of Sciences, Moscow, Russia \\ ${ }^{2}$ Moscow Institute of Physics and Technology, Dolgoprudny, Russia \\ *e-mail: pushkoval8@gmail.com
}

Key words: Populus; poplar; sex determination, de novo genome assembly; Nanopore; pure high-molecular-weight DNA

Motivation and Aim: Dioecy, i.e. the presence of males and females, is widespread among animals, but in plants, it is much less common - only 5\% of angiosperms are dioecious. The genus Populus is represented by dioecious species with XY or ZW sex-determination system and is characterized by polymorphic mechanisms of sex determination. $P . \times$ sibirica is one of the most common poplars in the Moscow region, but there is no genome assembly for it to date. Our aim was to achieve high-quality genome assemblies of male and female plants of $P$. x sibirica. We used a combination of the Oxford Nanopore Technologies (ONT) platform with long reads and the Illumina platform with high-accuracy reads.

Methods and Algorithms: Leaves were collected from male and female plants of $P$. x sibirica in the period of poplar bloom for the proper sex identification and immediately frozen in liquid nitrogen. We developed and optimized the protocol of obtaining pure high-molecular-weight genomic DNA from young poplar leaves and received DNA samples with approximately $50 \mathrm{~kb}$ length, A260/A280 of 1.7-2.0, and A260/A230 of 2.0-2.2. DNA libraries were prepared and sequenced on MinION (ONT) with FLO-MIN-106 R9.4 and FLO-MIN-106 R10 flow-cells (ONT) and HiSeq 2500 (Illumina) with a read length of 125+125 bp. Nanopore reads were processed using Guppy 3.6.1. Then, adapter sequences were removed using Porechop. Low-quality Nanopore and Illumina reads were filtered out and/or trimmed using Trimmomatic. Genome assemblies were performed using four assemblers: Flye 2.8, Raven 1.1.10, Shasta 0.5, and wtdbg2 2.5. Then, polishing was performed with Racon 1.4.3 using Nanopore reads and with POLCA from MaSuRCA 3.3.9 using Illumina reads.

Results: After sequencing on MinION, we received $22.3 \mathrm{~Gb}$ and $22.9 \mathrm{~Gb}$ with N50 about $20 \mathrm{~kb}$ for male and female plants respectively, while the use of HiSeq 2500 in 2x125 bp format resulted in 36 million reads and 52 million reads for the same plants. The best result was obtained for the Raven assembler: genome length was $474 \mathrm{Mb}$ with $\mathrm{N} 50$ of $0.33 \mathrm{Mb}$ for the male poplar and $495 \mathrm{Mb}$ with $\mathrm{N} 50$ of $0.32 \mathrm{Mb}$ for the female one. According to BUSCO, the completeness of genomes was $96.8 \%$ for the male and $97.9 \%$ for the female.

Conclusion: The combination of Nanopore and Illumina platforms allowed us to achieve a high-quality genome assembly of male and female plants of $P$. x sibirica that will provide an opportunity to compare the received data with the genome assemblies of genetically different poplars and to clarify the mechanism of poplar sex determination.

Acknowledgements: This work was funded by RFBR according to the research project 17-29-08036. 